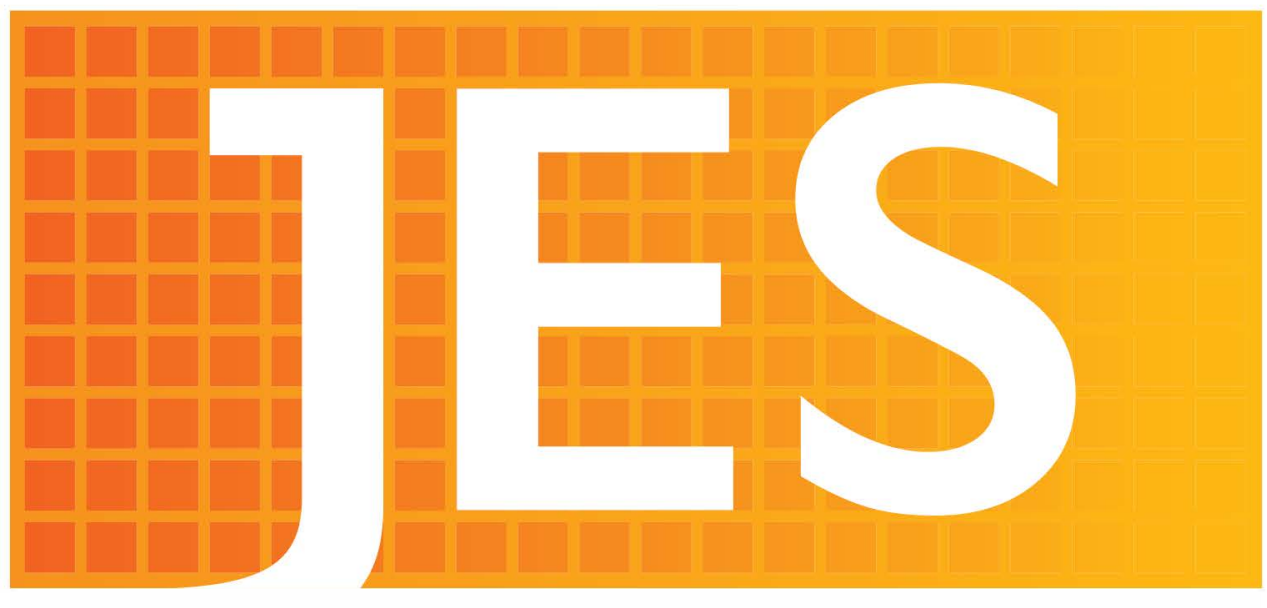

\title{
Journal of the Endocrine Society
}

AN OPEN ACCESS PUBLICATION

\section{ENDO 2019 ABSTRACTS} 101ST ANNUAL MEETING AND EXPO OF THE ENDOCRINE SOCIETY

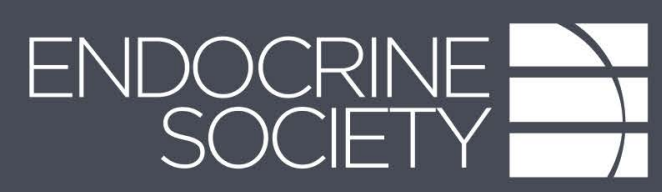




\section{Journal of the Endocrine Society ENDOCRINEY
SOCIETY}

\section{SUN-464 Trunk Fat Increase Is Prevented Both in}

\section{Patients Undergoing Long-Lasting Continuous $\mathrm{r}-\mathrm{hGH}$} Therapy and in Those Who Discontinued $\mathrm{r}-\mathrm{hGH}$ Compared to Untreated Patients: Results from Baseline Data of the MAGHD Study $\odot$

Maria Laura Monzani, MD, Elisa Magnani, MD, Chiara Diazzi, MD, PhD, Laura Volpi, Vincenzo Rochira, MD, PhD

Journal of the Endocrine Society, Volume 3, Issue Supplement_1, April-May 2019, SUN-464, https://doi.org/10.1210/js.2019-SUN-464

Published: 30 April 2019

Split View Cite Permissions Share $\mathbf{\nabla}$

\section{Abstract}

Background: Adult-onset growth hormone (GH) deficiency (AGHD) is related with alterations in body composition, increased abdominal and visceral adiposity, adverse change in lipid and carbohydrate metabolism, and reduction of bone mineral density (BMD). Aim: To compare body composition and biochemical/hormonal data among adult patients with AGHD referring to a single endocrinological center and grouped according to $\mathrm{r}-\mathrm{hGH}$ therapy. Methods: The Manage Growth Hormone Deficiency Study (MAGHD) is a prospective, single-center trial aiming to improve AGHD management through a smartphone app (MAGHD App: Manage AGHD) integrated with a software framework able to merge several kind of patients' daily data with clinical data collected in institutional databases. Up-to-now, a total of 74 subjects (26 Female, 48 Male, mean age: $56.6+14.8$ years) with AGHD, fulfilling the inclusion criteria, were enrolled in the study. According to r-hGH therapy, they were divided in 3 
groups: patients on long-term $\mathrm{r}$-hGH therapy (Group $1, \mathrm{n}=33$ ), patients previously treated with $\mathrm{r}-\mathrm{hGH}$, who had stopped the therapy at any time (Group 2, n=18), patients never treated (Group 3, n=23). Each patient has completed the baseline visit, including biochemical and hormonal measurements and data on BMD and body composition obtained by DXA. The nonparametric Kruskal-Wallis test was used for comparison among 3 groups and data are expressed as median. Results: Mean duration of AGHD disease of the entire cohort was 121.2+105.8 months; it was lower in Group 3 compared to both Group 1 and 2 ( $\mathrm{p}<0.05$ ). Waist circumference (Group 195 cm; Group 2 96,5 cm; Group 3109 cm) and trunk fat mass (Group 1539 g; Group 2525 g; Group 3595 g) were significantly higher in Group 3 compared to both Group 2 and 1 $(\mathrm{p}<0.05)$. Serum insulin, total fat mass, and total body fat $(\%)$ were significantly higher in Group 3 than Group 1 ( $\mathrm{p}<0.05$ ). IGF-1 and IGFBP3 were significantly higher in Group 1 compared to both Group 2 and 3 ( $\mathrm{p}<0.0001$ ). BMD, circulating lipids, and fasting glucose did not differ among the 3 groups. Conclusions: $r-h G H$ therapy seems to confer a long-lasting beneficial effect on body fat, especially trunk fat even after its discontinuation in AGHD patients, but not on metabolic parameters.

Acknowledgment: This clinical study is conducted thanks to the competitive assignment of an Independent Grant for Learning \& Change ("IGCL") Dissemination \& Implementation ("D\&I") by Pfizer Inc.

Issue Section: Neuroendocrinology and Pituitary

Copyright $\odot 2019$ Endocrine Society

This article has been published under the terms of the Creative Commons Attribution Non-Commercial, NoDerivatives License (CC BY-NC-ND; https://creativecommons.org/licenses/by-nc-nd/4.0/).

\section{Email alerts}

New issue alert 